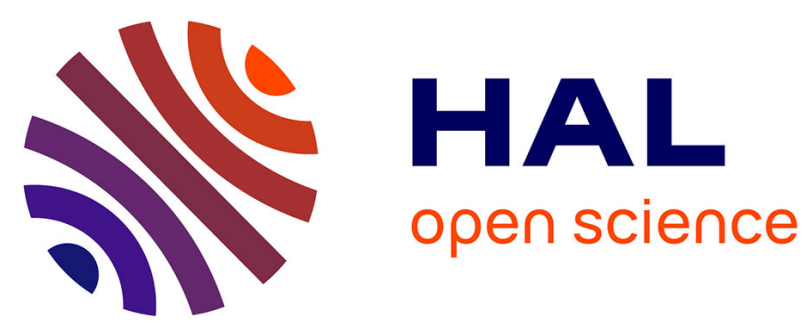

\title{
Compressed sensing for the extraction of atrial fibrillation patterns from surface electrocardiograms
}

Amina Ghrissi, Vicente Zarzoso

\section{To cite this version:}

Amina Ghrissi, Vicente Zarzoso. Compressed sensing for the extraction of atrial fibrillation patterns from surface electrocardiograms. EUSIPCO-2019, 27th European Signal Processing Conference, Sep 2019, A Coruna, Spain. 10.23919/EUSIPCO.2019.8902596 . hal-02486993

\section{HAL Id: hal-02486993 https://hal.science/hal-02486993}

Submitted on 21 Feb 2020

HAL is a multi-disciplinary open access archive for the deposit and dissemination of scientific research documents, whether they are published or not. The documents may come from teaching and research institutions in France or abroad, or from public or private research centers.
L'archive ouverte pluridisciplinaire HAL, est destinée au dépôt et à la diffusion de documents scientifiques de niveau recherche, publiés ou non, émanant des établissements d'enseignement et de recherche français ou étrangers, des laboratoires publics ou privés. 


\title{
Compressed sensing for the extraction of atrial fibrillation patterns from surface electrocardiograms
}

\author{
Amina Ghrissi and Vicente Zarzoso \\ Universite Côte d'Azur, CNRS, I3S Laboratory \\ Les Algorithmes, Euclide-B, 2000 route des Lucioles \\ 06903 Sophia Antipolis Cedex, France \\ Email: \{amina.ghrissi, vicente.zarzoso\} @ univ-cotedazur.fr
}

\begin{abstract}
The non invasive analysis of atrial fibrillation (AF) arrhythmia represents a challenge nowadays. The fibrillatory pattern of AF known as $f$-wave, is partially masked by the ventricular activity of the heartbeat in the surface electrocardiogram (ECG). Classical techniques aiming to extract the $f$-wave are based on average beat subtraction (ABS) or blind source separation (BSS). They present limitations in performance and require long ECG recordings as well as multi-channel records in the case of BSS. The originality of the present work consists in exploiting the sparsity of the atrial activity (AA) in the frequency domain to extract the full $f$-wave using a recent data acquisition technique called compressed sensing (CS). The present contribution takes a step forward in the extraction of the $f$-wave by exploiting the time rather than the space dimension. We intend to recover $A A$ signal with a variant of $C S$ where classical random sampling is replaced by block sampling scheme. Our breakthrough finding consists in the ability of our method to accurately extract the AA from a short ECG recording of just one heartbeat, with a normalized mean squared error of 0.150, which is unfeasible with ABS, BSS and other variants that require longer recordings.
\end{abstract}

\section{INTRODUCTION}

Atrial fibrillation (AF) is the most common sustained arrhythmia encountered in clinical practice. Held responsible of up to $25 \%$ of strokes, this cardiac condition is considered as the last great frontier of cardiac electrophysiology as it continues to puzzle cardiologists [1]. In order to better characterize this arrhythmia, scientists are interested in analyzing the pattern of $\mathrm{AF}$ noninvasively by extracting the $f$-waves of atrial activity (AA) from surface electrocardiogram (ECG) recordings [2]. The main classical cardiac signal processing tools for non invasive AA signal extraction are 1) average beat subtraction (ABS) technique [3], and 2) blind source separation (BSS) [4], [5]. To provide adequate performance, these techniques require records of sufficient length. Other techniques like interpolation are adapted to AA extraction [6]. The present work aims at overcoming the limitations of ABS and BSS. We intend to extract the AA and separate it from the dominating ventricular activity (VA, QRST complex), using compressed sensing (CS). This method takes advantage of the sparsity property of the fibrillatory signal in the frequency domain. To our knowledge, this is the first time CS is applied to noninvasive AA extraction. Our second contribution consists in introducing a block sampling scheme as opposed to the random sampling classically used in CS. We start by preliminary tests on simple signals in order to validate the implementation of our solution and its accuracy. The tests focus on the influence of the compression ratio on the quality of signal recovery added to the effect of block sampling. Second, CS is computed on synthetic $f$-waves. Third, we move to more realistic scenarios by applying CS to synthetic ECG signals with fibrillatory pattern. We compare CS performance to a state of the art $\mathrm{ABS}$ technique, adaptive singular value cancellation (ASVC). Comparison is mainly based on the quality of AA extraction, sensitivity to the length of the ECG recording and the computational cost.

This work is outlined as follows. Section II presents the extraction problem and summarizes classical cardiac signal processing tools used for $f$-wave estimation. Section III introduces CS in the context of AF ECG analysis. We study its mathematical definition and properties. Our results of CS signal reconstruction with different sampling approaches are presented in Section IV. Finally, the conclusions and perspectives of the work are presented in Section V.

\section{NoninVASIVE EXTRACTION OF AA IN AF}

\section{A. AF Diagnosis}

AF occurs when there exists an irregular and chaotic activation in the atrias, the upper chambers of the heart. Then instead of beating effectively to eject blood into the ventricles, the atrias start quivering or fibrillating, thus causing irregular fluctuations in the baseline. As a result, the ventricular rate becomes more rapid and irregular.

The ECG of patients suffering from AF is different from normal sinus rhythm. It is characterized by the absence of $P$ wave and the presence of $f$-waves visible in the TQ segments between consecutive beats. The AA occurs all throughout the recoding, but it is obscured by VA at each heartbeat [2]. Indeed, in the ECG we distinguish two kinds of intervals:

1) QT segments: both AA and VA happen simultaneously but the AA is masked by the QRST complex.

2) TQ segments: only AA takes place and it is perfectly known since the ventricles are inactive.

Our goal is estimating the AA in the QT segments in AF patients. 


\section{B. Atrial Activity Extraction}

We aim to extract the AA and separate it from the VA in ECG recordings. Several methods have been proposed for this task like ABS and BSS. Although partially successful, the latter methods present important limitations, as summarized below:

a) $A B S$ : Although multi-channel variants exist, $\mathrm{ABS}$ is adapted to single-lead ECGs [3]. This method computes a representative beat by synchronized averaging of the beats present in the recordings, thus requiring the recognition of beat morphology from the ECG. ABS is very sensitive to QRST wave variants and relies on high-quality cancellation templates that are in practice difficult to obtain from short single-lead ECG recordings.

ASVC of ventricular activity is a variant of ABS, developed in [3], that intends to overcome ABS inherent limitations. The method exploits the mutual information available in the set of ECG beats in order to extract the basis signal corresponding to the VA component. ASVC remains more robust than ABS in ECGs with variable QRST morphology and in the presence of ectopic beats. However, ASVC is limited by the number of beats to be processed and the length of ECG signals.

b) BSS: BSS is a statistical tool that consists in separating unobservable source signals from a set of observed mixture. Independent component analysis (ICA) belongs to BSS and is proven in [4] to accurately recover AA in AF. ICA reconstructs the unobservable independent sources of bioelectric activity which generate, through instantaneous linear mixing, a measurable set of signals. This approach exploits the spatial diversity provided by multi-lead ECG recordings with sufficient length to allow the estimation of higher order statistics with enough accuracy.

\section{Proposed Approach}

\section{A. Essence of Compressed Sensing}

Compressed sensing (CS) is a data compression paradigm that requires much less measurements than stated by the Nyquist rate. CS acquires a spread/dense signal $f$ accepting a sparse/compressible representation $x$, when expressed in the proper basis $\Psi$ :

$$
f=\Psi x .
$$

In practice we only observe a subset $\Omega \subset\{1, . ., N\}$ of size $|\Omega|=K<<N$, through a selection matrix $\Phi$ and the challenge is to recover the $N$-dimensional sparse signal $x$ from $y$ the $K$-dimensional measurement vector:

$$
y=\mathbf{R} \boldsymbol{\Phi} f=\mathbf{R} \boldsymbol{\Phi} \Psi x=\mathbf{U} x,
$$

$\mathbf{U}$ is a $(K \times N)$ matrix and $\mathbf{R}$, generally at random, is of the same size. $\mathbf{R}$ is the selection matrix.

CS recovers the sparse representation $x$ through suitable optimization tools [7]. The symbol $\|\cdot\|_{p}$ stands for $\ell_{p}$ norm:

$$
\min _{x \in R^{N}}\|x\|_{1} \quad \text { subject to } \quad y=\mathbf{U} x .
$$

From a linear algebra perspective, the reconstruction problem is ill posed, because $K<N$. CS guarantees the accurate reconstruction of the target signal through conditions and problem regularizations like the Restrictive Isomery Property (RIP) [7], [8].

\section{B. Compressed Sampling for Noninvasive Atrial Activity Ex- traction from the ECG}

Based on the physiological finding that the ventricular and atrial activities are uncoupled [1] and that the ECG is the sum of both, added to interferences with the activities of the surrounding organs, vessels and noise due to the acquisition system, we intend to extract the full AA from the observation of its signal in the TQ segment. In this context, we suggest to restrict the samples selection on the TQ segments from the ECG, where VA is null. We call this sampling scheme block sampling. The $f$-wave $\left(f_{A A}\right)$ is present all along the ECG recording $\left(f_{E C G}\right)$. The component $f_{A A}$ accepts a sparse representation in the frequency domain as in eq. (1). $f_{V A}$ is the interfering component present in QT intervals of ECG. $f_{A A}$ is present in both heart beating phases QT $\left(f_{Q T}\right)$ and TQ $\left(f_{T Q}\right)$ :

$$
\begin{gathered}
f_{E C G}=f_{A A}+f_{V A}, \\
f_{E C G}^{Q T}=f_{A A}^{Q T}+f_{V A}^{Q T}, \\
f_{E C G}^{T Q}=f_{A A}^{T Q} .
\end{gathered}
$$

The measurement signal is $y=f_{E C G}^{T Q}=f_{A A}^{T Q}$. The CS approach aims at reconstructing the full $f_{A A}$, including the unknown $f_{A A}^{Q T}$, from the only knowledge of $y$. We intend to use the samples of AA in TQ segments, which are perfectly known, to estimate/reconstruct, through CS, the unknown samples in QT intervals. Because the theoretical demonstration of this approach is a difficult task, the goal is to experimentally evaluate the block sampling scheme for AA signal recovery.

TABLE I

BLOCK SAMPLING OF ECG.

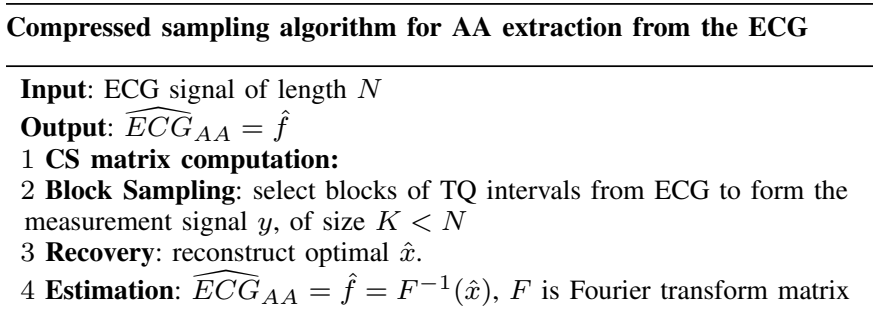

Block Sampling: Classical sampling process takes $K$ random measurements. Our proposed sampling scheme consists in taking blocks of the measurement of size $(p)$ alternated by blocks of unobserved signals of size $(q)$, as illustrated in Fig. 1. We call this scheme $(p, q)$ sampling. In practice, we measure the TQ and drop the QT intervals.

Our suggestion for block sampling needs to be experimentally validated and mathematically proven. We find in the literature a prior work that neglected parts of signal 


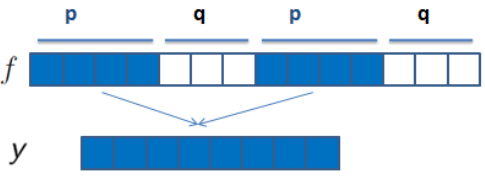

Fig. 1. $(p, q)$ sampling scheme

(image rows/columns) in the sampling, when applying CS to ultrasound images in [9]. This technique showed accurate signal reconstruction even under high compression ratios.

\section{EXPERIMENTAL RESULTS}

This section investigates the performance of CS technique for atrial signal $\left(f_{A A}\right)$ recovery in the case of patients with AF. In particular, interest is focused on the influence of block as opposed to random sampling in CS, as we intend to recover the $f_{A A}$ from measures restricted to the TQ segment in ECG recordings. Our solution is validated and tested on synthetic ECG signals containing AF patterns.

Complexity is added at each experiment level. The influence of the compression ratio on the accuracy of signal recovery is calculated. In order to quantify the recovery quality, the normalized mean squared error (NMSE) between the original and the reconstructed signals is computed. Then, signal is sampled by equal-sized-blocks of measurement instead of the standard random sampling protocol (block-sampling). First experiments are conducted on synthetic $f$-wave signals $\left(f_{\mathrm{AA}}\right.$ with AF pattern) generated according to the model of STRIDH and SÖRNMO'S model [11]. Second experiments are conducted on synthetic $f_{A A}$ superimposed to real ECG signals of VA $\left(f_{V A}\right)$ of subjects in sinus rhythm. The resulting signal approximates the ECG of an AF patient. Third, the CS approach is applied to complete ECG with AF patterns available in open source databases. Finally, the performance of CS is compared to that of ASVC in AA extraction from ECG. The experiments on CS are conducted with MATLAB software using the $\ell_{1}$-MAGIC toolbox [10].

\section{A. CS Recovery of isolated $f$-wave}

1) Genesis of Synthetic f-wave: We simulate synthetic $f$-wave according to STRIDH and SöRNMO's model [11]. The dynamics of AF have a modulated sawtooth-like shape, approximated by a sinusoid and $(M-1)$ harmonics. The sawtooth amplitude is given by a time-varying amplitude $a_{l, i}$, a phase $\theta(n)$ and cycle length, thus introducing a non-stationary behavior:

$$
f_{l}(n)=\sum_{i=1}^{M} a_{l, i}(n) \sin (i \theta(n)), \quad n=1, . ., N .
$$

The AA $\left(f_{l}\right)$ is observed in the $l^{\text {th }}$ lead along $N$ samples. We recall $\theta$ depends on the fundamental frequency of this pattern $h_{0} \in[3,9] \mathrm{Hz}$. We simulate a paroxysmal AF pattern with $M=5$, main amplitude $a_{1}=150 \mu V\left(a_{l, i}\right.$ depends on $\left.a_{l}\right)$ and dominating frequency $h_{0}=6 \mathrm{~Hz}$ as in [11].
2) Influence of Block Size and CR on the NMSE: We perform bare AF signal recovery ( $f=f$-wave) for different combinations of $p$ and $q$ yielding the compression ratio (CR):

$$
\mathrm{CR}=\frac{p}{p+q} \text {. }
$$

After sampling, the recovery is assessed by normalized mean squared error (NMSE) between the original signal and the recovered one:

$$
\operatorname{NMSE}(f)=\frac{\|f-\hat{f}\|_{2}{ }^{2}}{\|f\|_{2}{ }^{2}} .
$$

Fig. 2 shows the curves of the NMSE versus different combinations of $p$ and CR in logarithmic scale. The experiments are conducted on a 6 second recording.

We recall that the physiological duration of QT and TQ segments are approximately in the ranges of $[366,383]$ and $[422,481] \mathrm{ms}$, respectively in normal heart rhythm [12]. Thus the CR in AA extraction from ECG is approximated by the ratio $\frac{\mathrm{TQ}}{\mathrm{TQ}+\mathrm{QT}}$.

$$
\mathrm{CR} \simeq \frac{\mathrm{TQ}}{\mathrm{TQ}+\mathrm{QT}} \in[0.4,0.6]
$$

For $\mathrm{CR} \in[0.4,0.6]$, the NMSE resulting from block sampling, for all values of $p$ are in $[-10,0](\mathrm{dB})$. For values of CR close to 0.9 and 1 , it gives low NMSE and the curves tend to $-\infty$ in $(\mathrm{dB})$.

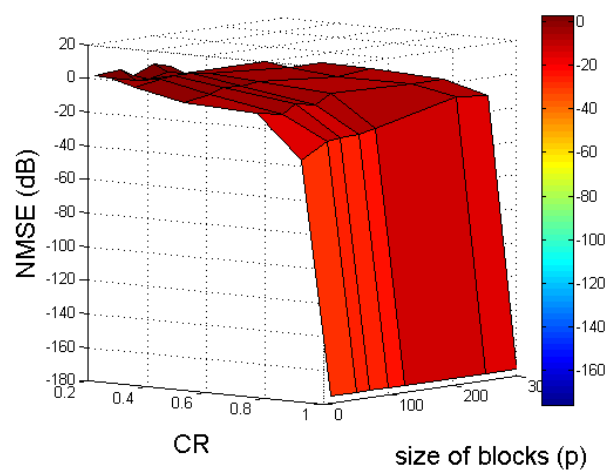

Fig. 2. NMSE (dB) of reconstructed synthetic atrial signal for block sampling for different combinations of $p$ and CR $(\mathrm{dB})$.

\section{B. CS Recovery of $f$-wave Corrupted by VA}

1) Genesis of Synthetic ECG with Fibrillatory Pattern: Similarly to the process of data synthesis in [1], we suggest to generate synthetic ECG contaminated by AF pattern by superimposing synthetic $f$-waves generated by the above STRIDH and SöRNMO's model [11] to a VA signal containing only QT complex. The $f_{V A}$ is generated from a surface ECG of a healthy subject after the following steps:

1) Acquire sinus rhythm surface ECG data of healthy adults from the PTB Diagnostic ECG Database (PTB) [13], at a sampling rate of $1 \mathrm{KHz} .2$ ) Denoise the data and remove baseline wander and powerline interference, we preprocess it 
using a forward-backward bandpass type-II Chebyshev IIR filter with cut-off frequencies of $0.5 \mathrm{~Hz}$ and $30 \mathrm{~Hz}$. 3) Manually delete $P$-waves as they explicitly reflect the AA. The $P$-waves are segmented and then suppressed by spline interpolation between their onset and offset points.

We focus our experiments on ECG signals acquired from the $V 1$ lead because the AA is significantly clearer and measurable in the chest position where $V 1$ lead is placed.

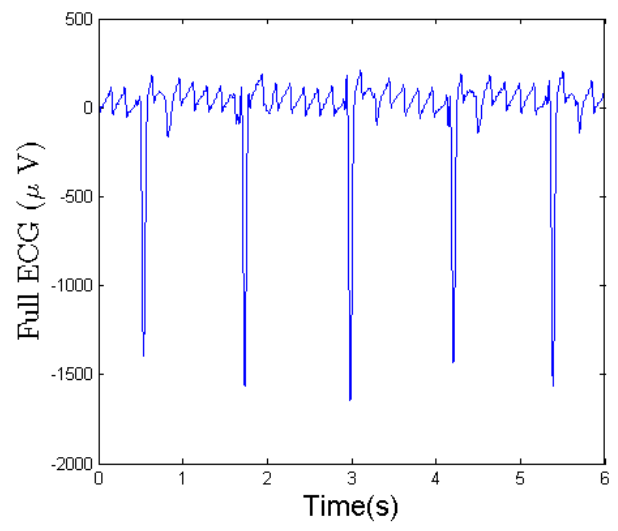

Fig. 3. Synthetic ECG with fibrillatory pattern.

\section{Extraction of $f$-wave from Full Synthetic ECG}

1) Long recording: Fig. 4 shows that both algorithms, CS and ASVC succeed in estimating a fibrillatory pattern in both locations of QT and TQ segments. Therefore, the accuracy of ASVC outperforms CS with an NMSE of $f_{A A}$ recovery in TQ segments $\left(f_{A A}^{T Q}\right)$ equal to 0.406 compared to 1.4. This accuracy is also better in recovering non measured segments $\operatorname{NMSE}\left(f_{A A}^{Q T}\right)$. The algorithm running time takes 0.5 $\mathrm{h}$ compared to $1.05 \mathrm{~s}$ in the case of ASVC.

Recovery of isolated $f$-wave: We use the same sampling scheme, as previously explained, to recover the isolated $f$ wave using CS solution. The overall recovery quality is acceptable with an $\operatorname{NMSE}\left(f_{A A}\right)=0.201$. Also, the recovery error is near zero in the sampled data (TQ segments), $9 \times 10^{-27}$.

However, ASVC computes a synchronous mean of the heart beats then subtracts the average QRST complex from ECG. For this purpose this techniques operates on recordings having full QRST complexes, otherwise it will try to complete the missing parts of these complexes, if missing in the end of the signal. In this context, when we recompute ASVC on 6 $\mathrm{s}$ recording with non complete QT segment, the solution fails to extract the AA in this segment and it recovers the VA also, as shown in Fig. 5. This is because the last beat is not taken into account in the QRST cancellation process.

2) Short recording: We have seen in the previous experiment that the accuracy of ASVC technique outperforms CS and that its computational cost is significantly less important, regarding the running time and the complexity of solving an optimization convex program in CS compared to synchronously averaging beats in ASVC. However, we discover:

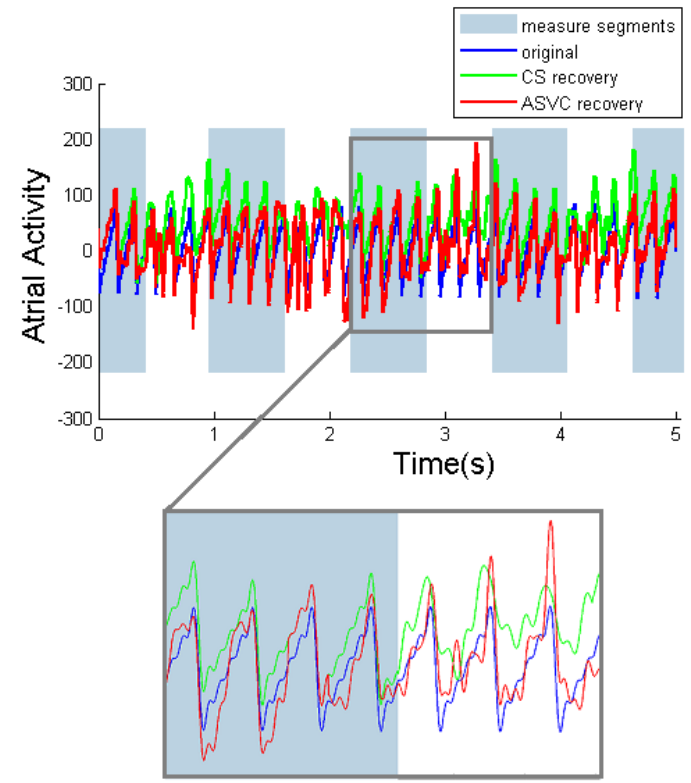

Fig. 4. Extraction of $f$-wave from synthetic ECG with CS (green) and ASVC (red) vs. the original fibrillatory signal (blue).

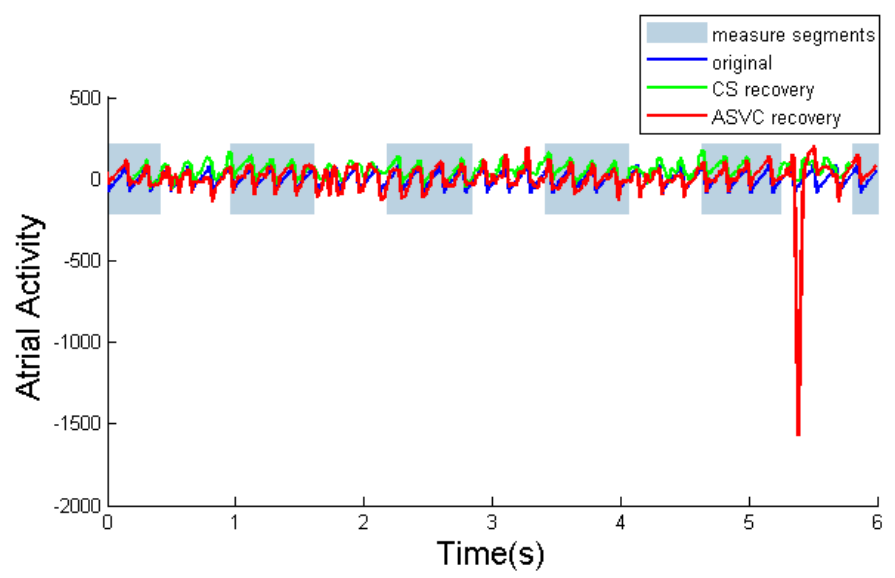

Fig. 5. Extraction of $f$-wave from synthetic ECG (6 s recording) with CS (green) and ASVC (red) vs. the original fibrillatory signal (blue).

One-Heartbeat ECG: CS is able to recover the signal from a short recording of almost $1 \mathrm{~s}$, containing 1 heartbeat in average, as illustrated in Fig. 6. However, ASVC is not designed to work in this case, as its performance is said to be acceptable for an ECG recording containing at least 10 beats [3], that is almost $15 \mathrm{~s}$ (15000 samples for a high resolution sampling frequency $f_{s}=1 \mathrm{Khz}$ ).

Bias-corrected CS or Corrected CS (CCS): We notice in the previous experiment (One-Heartbeat ECG) that although the recovered AA with CS mimics almost perfectly the original one, its NMSE is important $\left(\operatorname{NMSE}\left(f_{A A}\right)=1.797\right)$. When observing Fig. 6 , we notice the $\hat{f}_{A A}$ seems biased by a trend and its overall shape is accurate. To suppress the bias, we suggest to subtract from the restriction of $\hat{f}_{A A}$ to each 
segment $\left(\hat{f}_{A A}^{\text {segment }_{i}}\right)$ its mean. The $\left(\text { segments }_{i}\right)_{i=1 . .3}$ represent the samples of intervals TQ and QT alternatively. We notice discontinuity between the $\hat{f}_{A A}^{\text {segment }} i^{\prime} s$ because the means of the different signal portions are different. To handle this issue, we suggest to correct the CS technique by subtracting from $\hat{f}_{A A}$ its full mean $\left(\operatorname{mean}\left(\hat{f}_{A A}\right)\right)$. The overall $\operatorname{NMSE}\left(f_{A A}\right)$ after bias correction is equal to just 0.150 . The performance of CS

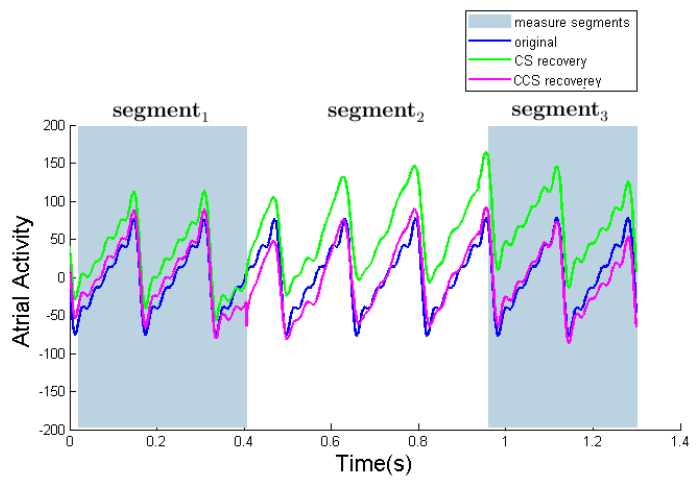

Fig. 6. Extraction of $f$-wave from synthetic ECG (1-heartbeat recording) with bias-corrected CS (magenta) vs. standard CS (green) vs. the original fibrillatory signal (blue).

TABLE II

NMSE OF $f$-WAVE EXTRACTION FROM SYNTHETIC AF ECG WITH BIAS-CORRECTED CS FROM A 1-HEARBEAT ECG.

\begin{tabular}{|c|c|c|c|}
\hline Algorithm & $\operatorname{NMSE}\left(f_{A A}^{\text {segment }_{1}}\right)$ & $\operatorname{NMSE}\left(f_{A A}^{\text {segment }_{2}}\right)$ & $\operatorname{NMSE}\left(f_{A A}^{\text {segment }_{3}}\right)$ \\
\hline CCS & 0.127 & 0.194 & 0.115 \\
\hline
\end{tabular}

is improved by mean subtraction for each TQ and QT segment.

\section{CONCLUSIONS}

The present contribution, has put forward a new technique for extracting the fibrillatory pattern of $\mathrm{AF}$ from surface ECG based on the CS paradigm. To our knowledge, this is the first time $\mathrm{CS}$ is applied to AA extraction. We suggest to exploit the fact that the AA in the ECG has a sparse frequency distribution, therefore it could be recovered using $\mathrm{CS}$. It is proposed to replace the classical random sampling of the measurement signal in CS by a block sampling that selects only VA-free segments from ECG and neglects QRS complexes. Thus, recovering only the full AA.

The results show that the accuracy of CS is lower than ASVC in terms of NMSE when performing on full long recordings. However, a breakthrough finding is the ability of CS to extract AA from a short ECG recording containing only one heartbeat, which is impossible with ASVC, that is said to perform well for significantly longer recordings of at least 10 heartbeats. Based on the observation that CS performs well on short recordings, it is adapted to an online process, where it estimates the AA beat-by-beat from the ECG. This way, our CS approach handles better long recordings. On the other hand, ASVC performs heartbeats detection and classification before the extraction process thus being very sensitive to the heartbeats morphology, location and duration. A biascorrected variant of our method proves to be more accurate with an $\operatorname{NMSE}\left(f_{A A}\right)$ equal 0.150 versus 1.797 in classical CS technique. Finally, we asses the influence of the number of heartbeats on the accuracy of CS recovery. A major drawback of CS is its high computational cost. Despite these apparent limitations, experimental results are encouraging. Further work should aim at justifying mathematically the validity of block sampling according to the RIP and validating our method on a full AF ECG database. The sensitivity of our approach to the heartbeats morphology should also be checked.

\section{ACKNOWLEDGMENT}

A. Ghrissi is partially funded by the Labex scholarship programUCN@Sophia. V. Zarzoso is a member of the Institut Universitaire de France. We are grateful to Olivier Meste for his insightful remarks

\section{REFERENCES}

[1] L. N. Ribeiro, A. RHidalgo-Muñoz, and V. Zarzoso. "Atrial signal extraction in atrial fibrillation electrocardiograms using a tensor decomposition approach," In Engineering in Medicine and Biology Society (EMBC), IEEE, pp. 6987-6990. [37th Annual International Conf. of the IEEE].

[2] J. Malmivuo and R. Plonsey. Bioelectromagnetism: Principles and Applications of Bioelectric and Biomagnetic Fields. Oxford University Press, USA, 1995.

[3] R. Alcaraz and J. J. Rieta. "Adaptive singular value cancelation of ventricular activity in single-lead atrial fibrillation electrocardiograms," Physiological measurement, vol. 29, p. 1351, 2008.

[4] J. J. Rieta, F. Castells, C. Sanchez, V. Zarzoso, and J. Millet. "Atrial activity extraction for atrial fibrillation analysis using blind source separation," IEEE Transactions on Biomedical Engineering, vol. 51, pp. 1176- 1186, 2004.

[5] V. Zarzoso, O. Meste, P. Comon, D. G. Latcu, and N. Saoudi. "Noninvasive cardiac signal analysis using data decomposition techniques," In P. Kornprobst, O. Faugeras, and F. Cazals, editors, Modeling in Computational Biology and Biomedicine, chap. 3, pp. 83-116. Springer, 2013.

[6] L. Sörnmo, A. Petrènas, P. Laguna and V. Marozas. BookChapter: "Extraction of $\mathrm{f}$ waves. in Atrial Fibrillation from an Engineering Perspective," chap 5 pp. 137-220. Springer, 2018 .

[7] E. Candès and M. B. Wakin. "An introduction to compressive sampling," IEEE signal processing magazine, vol. 25, p. 21, 2008.

[8] E. Candès and J. Romberg. "Sparsity and incoherence in compressive sampling," Inverse problems, vol. 23, p. 969, 2007.

[9] C. Quinsac, A. Basarab, and D. Kouamé. "Frequency domain compressive sampling for ultrasound imaging," Advances in Acoustics and Vibration, vol. 2012, 2012.

[10] E. Candès and J. Romberg. 11-magic: Recovery of sparse signals via convex programming," vol. 4, p. 14, 2005.

[11] M. Stridh and L. Sörnmo. "Spatiotemporal QRST cancellation techniques for analysis of atrial fibrillation," IEEE Transactions on Biomedical Engineering, vol. 48, pp. 105-111, 2001.

[12] AA. Fossa, T. Wisialowski, K. Crimin, E. Wolfgang, JPH. Couderc, M. Hinterseer, S. Kaab, W. Zareba, F. Badilini, and N. Sarapa. "Analyses of dynamic beat-to-beat QT-TQ interval (ECG restitution) changes in humans under normal sinus rhythm and prior to an event of torsades de pointes during QT prolongation caused by sotalol," Annals of noninvasive electrocardiology, vol. 12, pp. 338348, 2007.

[13] AL. Goldberger, L. Amaral, L. Glass, JM. Hausdorff, PCh. Ivanov, RG. Mark, JE. Mietus, GB. Moody, C-K. Peng, and HE. Stanley. Physiobank, physiotoolkit, and physionet. Components of a New Research Resource for Complex Physiologic Signals., Circulation 101(23):e215-e220, 2000. 